\title{
Reliable inference of light curve parameters in the presence of systematics
}

\author{
Neale P. Gibson ${ }^{1,2}$ \\ ${ }^{1}$ European Southern Observatory, Karl-Schwarzschild-Str. 2, 85748 Garching bei München, \\ Germany \\ ${ }^{2}$ Astrophysics Research Centre, School of Mathematics and Physics, Queens University \\ Belfast, Belfast BT7 1NN, UK \\ email: n.gibson@qub.ac.uk
}

\begin{abstract}
Time-series photometry and spectroscopy of transiting exoplanets allow us to study their atmospheres. Unfortunately, the required precision to extract atmospheric information surpasses the design specifications of most general purpose instrumentation. This results in instrumental systematics in the light curves that are typically larger than the target precision. Systematics must therefore be modelled, leaving the inference of light-curve parameters conditioned on the subjective choice of systematics models and model-selection criteria. Here, I briefly review the use of systematics models commonly used for transmission and emission spectroscopy, including model selection, marginalisation over models, and stochastic processes. These form a hierarchy of models with increasing degree of objectivity. I argue that marginalisation over many systematics models is a minimal requirement for robust inference. Stochastic models provide even more flexibility and objectivity, and therefore produce the most reliable results. However, no systematics models are perfect, and the best strategy is to compare multiple methods and repeat observations where possible.
\end{abstract}

Keywords. methods: data analysis, techniques: spectroscopic, planetary systems:

\section{Introduction}

Transiting exoplanets allow us to study their compositions and atmospheres via wavelength dependent variations in their light curves using transmission and emission spectroscopy, and significant progress has been made since the first detection of an exoplanet's atmosphere (Charbonneau et al. 2002). These techniques require exquisite temporal stability in the light curves in order to measure $\sim 10^{-4}$ or smaller variations in the transit or eclipse depth as a function of wavelength (e.g. Seager \& Sasselov 2000; Brown 2001). Despite the enormous challenges and huge scientific reward, there are still no dedicated instruments for the study of transiting exoplanet atmospheres. Consequently, we have been using common-user instruments for many years that were not designed with this level of precision in mind. While we can easily collect sufficient photons to reach $\sim 10^{-4}$ precision, instrumental systematics limit the achievable precision in all of our observations. Our ability to reliably model these systematics is therefore of fundamental importance to our understanding of exoplanet atmospheres, and we are yet to reach a consensus in the community on how to address this.

Typically, systematics are modelled as a function of auxiliary inputs. These are measurements obtained simultaneously with the exoplanet time-series that are (thought to be) related to the underlying cause of the systematics, e.g. position of the image/spectral trace on the detector, width of the instrumental profile, or specifically for the Hubble Space Telescope (HST), its orbital phase. There are multiple approaches to constructing a systematics model. This short review aims to place the most commonly-used models 
in context, comparing the use of deterministic and stochastic models, and outlining the best practices for modelling exoplanet light curves in the presence of systematics. It is largely based on Gibson (2014), to which the reader is referred for more details. Here, I only focus on general systematics models, and do not consider instrument specific cases.

\section{Modelling instrumental systematics}

Systematics models fall into two main classes: deterministic and stochastic processes. A deterministic model is where the systematics are defined as a simple function of inputs, ranging from the simple case of a polynomial of time, to more complex functions of multiple inputs. Stochastic processes allow us to use probability distributions over functions, and offer much more flexibility. Gibson (2014) compares these models and tests their applicability on simulated transit light curves. Here, I summarise the models and results.

\subsection{Arbitrary systematics models}

Deterministic models are the simplest to construct, and the default choice is a linear model of the auxiliary inputs. With enough inputs (or higher order terms), these often appear to accurately model the systematics in the light curves, leaving the residuals adequately 'whitened'. However, many different systematics models can appear to account for instrumental systematics, yet produce significantly different results. Gibson et al. (2011) discussed this in the context of HST transmission spectra. In short, such methods produce spurious results that depend on arbitrary human choices, and should be avoided.

\subsection{Model selection}

Where many different systematics can be constructed, Bayesian model selection should allow us to select the best model, and remove human bias in this process. Indeed, this is the approach most widely used in the exoplanet community today (e.g. Sing et al. 2011, Nikolov et al. 2014). In general, we should calculate the Bayesian evidence of each model, however, this is often difficult to compute, and simple model selection criteria are used to estimate it. The most popular of these is the Bayesian Information Criterion (BIC), which is based on a simple addition of a term to the maximum likelihood value (or equivalently minimised $\chi^{2}$ ) which penalises the complexity of the model (via the number of free parameters).

Given its widespread use, Gibson (2014) tested the validity of this approach, by simulating tens of thousands of transit light curves with injected systematics, and attempted to recover the transit depth with uncertainties using a family of systematics models, including the correct model. This found that simple model selection routinely results in underestimated uncertainties in the extracted light curve parameters (in the case of the BIC, typically 100\%!). Full calculation of the Bayesian evidence fared better, but still did not provide reliable statistics. This means that model selection does not work in practice, and we should be careful when interpreting results extracted in this way.

\subsection{Marginalisation over many models}

The above result might seem surprising. However, when performing model selection, we are neglecting that there is uncertainty associated with the choice of systematics model, as well as the parameters of a specific model. In order to account for this, we can marginalise over many systematics models rather than force ourselves into selecting a single one. This is straightforward in practice, and requires minimal additional computation on top of model selection. In a nutshell, the evidence or information criteria for each model can be converted into a probability for each model (generally assuming uniform priors 
on the models), and the light curve parameters can be determined from a weighted average (based on these probabilities) of the marginalised posterior distribution for each individual model. Gibson (2014) shows that this provides much more reliable statistics of the recovered light curve parameters than model selection. Where the evidence clearly prefers one model over the others, this process reduces to model selection.

\subsection{Stochastic models}

Marginalisation over many models (and model selection) assumes that one of the systematics models tested is the correct one. Stochastic processes offer an even more flexible alternative to modelling instrumental systematics, and largely avoids this problem. These were introduced by Gibson (2012) in the form of Gaussian processes (GPs). Rather than defining our model as a specific function of the inputs (i.e. a deterministic process), a GP allows us to place a probability distribution over a class of functions. For example, using the 'squared exponential' GP defines a distribution over any smooth function of the inputs, without needing to specify the exact form of the function itself. GPs are also intrinsically Bayesian, therefore penalise complexity in much the same way as model selection/marginalisation, although do so over an effectively infinite number of models. GPs can therefore marginalise out our ignorance of the unknown form of the systematics, and therefore provides a robust measurement of light curve parameters. Gibson (2014) tested the use of GPs on tens of thousands of simulated light curves (injected with deterministic and stochastic forms of systematics), and confirmed that they are the most reliable method to model systematics among those discussed here.

\section{Conclusions}

The method used to model instrumental systematics in exoplanet light curves is of central importance to our understanding of exoplanet atmospheres, and great care is needed to construct useful models. However, the choice of systematics model will always contribute to the error budget, and is usually neglected. This must be taken into account by integrating out our ignorance of the form of the systematics model. I recommend the use of marginalisation over many models when using deterministic models; however, stochastic processes will over an even greater degree of flexibility and objectivity, and it is even possible to marginalise over many stochastic models for very complex problems.

Of course, no systematics models are perfect, and a combination of the above methods is the best approach, tested alongside instrument-specific models and preferably with repeated observations. However, the quality of extracted spectra will always be limited by the systematics, where uncertainty in model parameters and model selection always contribute to the error budget. To make the next leap in our understanding of exoplanets, dedicated instrumentation is needed to minimise the systematics in our observations.

\section{References}

Brown, T. M. 2001, ApJ, 553, 1006

Charbonneau, D., Brown, T. M., Noyes, R. W., \& Gilliland, R. L. 2002, ApJ, 568, 377

Gibson, N. P., Pont, F., \& Aigrain, S. 2011, MNRAS, 411, 2199

Gibson, N. P., Aigrain, S., Roberts, S. et al. 2012, MNRAS, 419, 2683

Gibson, N. P. 2014, MNRAS, 445, 3401

Nikolov, N. et al. 2014, MNRAS, 437, 46

Seager, S. \& Sasselov, D. D. 2000, ApJ, 537, 916

Sing, D. K. et al. 2011, MNRAS, 416, 1443 